Short Communication

\title{
Preparation of Three-dimensional Mesoporous Carbon Electrode Materials as Electrocatalysts for Hydrogen Evolution Reaction
}

\author{
Zhengping Zhao ${ }^{1,2}$, Jiahao Wang ${ }^{2}$, Xuan $\mathrm{Yu}^{1}$, Haojie $\mathrm{Li}^{1}$, Leiting Han ${ }^{1}$, Zhiquan Wang ${ }^{1}$, Dan Qiao ${ }^{1}$, \\ Mingqiang Zhong ${ }^{2}$ and Xiufang Chen ${ }^{3 *}$ \\ ${ }^{1}$ Zhijiang College, Zhejiang University of Technology, Hangzhou 310014, China \\ ${ }^{2}$ College of Materials Science and Engineering, Zhejiang University of Technology, Hangzhou \\ 310014, China \\ ${ }^{3}$ National Engineering Lab for Textile Fiber Materials \& Processing Technology, College of Materials \\ Science and Engineering, Zhejiang Sci-Tech University, Hangzhou 310018, China \\ *E-mail: sjzhaolei@163.com
}

doi: $10.20964 / 2021.04 .45$

Received: 30 November 2020 / Accepted: 26 January 2021 / Published: 28 February 2021

\begin{abstract}
In order to improve the electrochemical performance of hydrogen evolution electrode, this paper presents a new method of the preparation of the negative electrode materials with high activity of hydrogen evolution. Based on the principle of self-assembly, polyphosphazene microspheres were prepared by precipitation polymerization and carbonized in $\mathrm{N}_{2}$ atmosphere to form blank porous carbon spheres with excellent electrical conductivity, good physical/chemical stability and outstanding absorption performance. Then NiO/Ni@C-PZS were prepared by chemical precipitation method and carbonization loading method, and labeled as sample A and sample B, respectively. It was observed that, compared with the blank carbon spheres, sample A and sample B had higher hydrogen evolution activities by electrochemical measurement. Under the same scan rate, the capacitance performance of sample A and sample B are improved significantly and have obvious redox peaks. It can be found from the LSV that the hydrogen evolution overpotential of sample A is $0.4 \mathrm{~V}$ (vs. RHE), and the $\eta$ of sample A is basically unchanged after 100 cycles, which indicates that sample A has good cycle stability. The result that the tafel slope of sample B is only $81 \mathrm{mV} \cdot \mathrm{dec}^{-1}$, far lower than the $122 \mathrm{mv} \cdot \mathrm{dec}^{-1}$ of C-PZS, indicating that the existence of $\mathrm{NiO} / \mathrm{Ni}$ plays an important role in hydrogen evolution activity of $\mathrm{NiO} / \mathrm{Ni} @ \mathrm{C}-\mathrm{PZS}$ electrodes and the hydrogen evolution reaction is consistent with the Volmer-Heyrovsky mechanism.
\end{abstract}

Keywords: Hydrogen evolution electrode, Porous carbon material, Electrocatalysis 


\section{FULL TEXT}

(C) 2021 The Authors. Published by ESG (www.electrochemsci.org). This article is an open access article distributed under the terms and conditions of the Creative Commons Attribution license (http://creativecommons.org/licenses/by/4.0/). 